\title{
Similar Constructing Method for Solving the Boundary Value Problem of the Composite First Weber System
}

\author{
Dong Xiaoxu ${ }^{1, *}$, Li Shunchu ${ }^{1}$, Gui Dongdong ${ }^{2}$, Pu Jun ${ }^{1}$, Li Huichun ${ }^{3}$ \\ ${ }^{1}$ School of Mathematics and Computer Engineering, Xihua University, Chengdu, China \\ ${ }^{2}$ Beijing Dongrunke Petroleum Technology Co., Ltd., Beijing, China \\ ${ }^{3}$ Geological Research Department of the Fourth Oil Production Plant of DaGang Oilfield Company, Tianjin, China \\ *Corresponding author: dongxiaoxu1028@163.com
}

Received July 21, 2013; Revised August 16, 2013; Accepted September 25, 2013

\begin{abstract}
In this paper, we solve a class of boundary value problems of the composite first Weber system. In the process of solving the problem, first of all, we introduce functions of guide solution. Secondly, we constructive similar kernel functions. Finally, solutions with a form of continued fraction product to boundary value problem of the composite first Weber system are obtained by assembling coefficients of the non-homogeneous left boundary condition, functions of guide solution, coefficients of two connection conditions and similar kernel functions. Then a new method is obtained for solving the composite boundary value problem-Similar Constructing Method (shortened as SCM). This method is not only simple and effective for solving the complicated boundary value problem of differential system, but also is a kind of innovative idea.
\end{abstract}

Keywords: boundary value problem, composite Weber system, similar constructing method, similar kernel function, function of guide solution

Cite This Article: Dong Xiaoxu, Li Shunchu, Gui Dongdong, Pu Jun, and Li Huichun, "Similar Constructing Method for Solving the Boundary Value Problem of the Composite First Weber System." American Journal of Applied Mathematics and Statistics 1, no. 4 (2013): 76-82. doi: 10.12691/ajams-1-4-5.

\section{Introduction}

As is known to all, a lot of mathematical models, which are abstracted from engineering technical problems such as heat conduction of composite material and seepage in composite medium, can be attributed to models for solving boundary value problems of composite ordinary differential system. Thus acquiring the solution to the boundary value problem is very important for solving practical engineering problems.

At the beginning of this century, the thought of similar structure of the solution began to form. Li Shunchu and others studied solutions to some second order linear homogeneous ordinary differential equations [1-8], partial differential equations which can be transformed into ordinary differential equations [9,10,11,12] and some seepage equations in oil and gas reservoir engineering [1326] respectively. Some gratifying results have been achieved that solutions to boundary value problems of differential equations can be expressed as a continued fraction or continued fraction product form (i.e. solutions have the similar structure) by introducing the similar kernel functions, while structure of solutions only are associated with the non-homogeneous boundary condition, and similar kernel functions are associated with the governing equation and other homogeneous boundary condition.
Based on the above study, this paper will study a class of boundary value problems of the composite first Weber system. Firstly, we structure functions of guide solution of left region by using two linear independent solutions to governing equation of left region of the boundary value problem and structure functions of guide solution of right region by using two linear independent solutions to governing equations of right region of the boundary value problem respectively. Secondly, we structure the similar kernel function of right region by using functions of guide solution of right region and coefficients of the right homogeneous boundary condition and structure the similar kernel function of left region by using functions of guide solution of left region, coefficients of two connection conditions and the right similar kernel function. Finally, the solution of left region to boundary value problem is obtained by assembling coefficients of the left non-homogeneous boundary condition and the similar kernel function of left region. The solution of right region to boundary value problem is obtained by assembling coefficients of the left non-homogeneous boundary condition, similar kernel functions of left and right region, coefficients of two connection conditions and functions of guide solution of left region.

In this paper, boundary value problem of the composite first Weber system is studied as follows: 


$$
\left\{\begin{array}{l}
z_{1}^{\prime \prime}+\left(2 n_{1}+1-x^{2}\right) z_{1}=0 \\
z_{2}^{\prime \prime}+\left(2 n_{2}+1-x^{2}\right) z_{2}=0 \\
{\left[E z_{1}+(1+E F) z_{1}^{\prime}\right]_{x=a}=D} \\
\left.z_{1}\right|_{x=c}=\left.\lambda z_{2}\right|_{x=c} \\
\left.z_{1}^{\prime}\right|_{x=c}=\left.\mu z_{2}^{\prime}\right|_{x=c} \\
{\left[M z_{2}+N z_{2}^{\prime}\right]_{x=b}=0}
\end{array}\right.
$$

where $D 、 E 、 F 、 M 、 N 、 a 、 b 、 c$ are constant, $n_{i} \geq 1,0<a<c<b, M^{2}+N^{2} \neq 0$.

\section{Preliminary Knowledge}

\subsection{Lemma 1}

With the variable substitutions $z_{i}=e^{-\frac{1}{2} x^{2}} y_{i}(i=1,2)$, the first Weber equations $z_{i}^{\prime \prime}+\left(2 n_{i}+1-x^{2}\right) z_{i}=0(i=1,2)$ can be transformed into Hermite equations of order $2 n_{i}(i=1,2)$ as follows [27].

$$
y_{i}^{\prime \prime}-2 x y_{i}^{\prime}+2 n_{i} y_{i}=0(i=1,2)
$$

\subsubsection{Proof}

By taking variable substitution $z_{i}=e^{-\frac{1}{2} x^{2}} y_{i}$ for the first Weber equation and calculating first-order derivative and two-order derivative of $z_{i}=e^{-\frac{1}{2} x^{2}} y_{i}$ to $x$ (i.e. $\left.z_{i}^{\prime}=e^{-\frac{1}{2} x^{2}}\left(y_{i}^{\prime}-x y_{i}\right), z_{i}^{\prime \prime}=e^{-\frac{1}{2} x^{2}}\left[y_{i}^{\prime \prime}-2 x y_{i}^{\prime}+\left(x^{2}-1\right) y_{i}\right]\right)$, the first Weber equation can be transformed into the equation as follows:

$$
y_{i}^{\prime \prime}-2 x y_{i}^{\prime}+2 n_{i} y_{i}=0(i=1,2)
$$

Where, Eq. (2) is the Hermite equation of order $2 n_{i}$.

\subsection{Lemma 2}

General solution to the first Weber equation can be expressed as [27]:

$$
z_{i}=e^{-\frac{1}{2} x^{2}}\left[A_{i} H_{n_{i}}(x)+B_{i} G_{n_{i}}(x)\right](i=1,2)
$$

where $A_{i}, B_{i}$ are arbitrarily real constants, and $H_{n_{i}}(\cdot), G_{n_{i}}(\cdot)$ are the first and the second class of Hermite functions of order $n_{i}$.

\subsubsection{Proof}

General solution to the Hermite equation can be expressed as:

$$
y_{i}=A_{i} H_{n_{i}}(x)+B_{i} G_{n_{i}}(x)(i=1,2) .
$$

According to the lemma 1 , we let $z_{i}=e^{-\frac{1}{2} x^{2}} y_{i}$, and then general solution to the first Weber equation can be obtained as follows:

$$
z_{i}=e^{-\frac{1}{2} x^{2}}\left[A_{i} H_{n_{i}}(x)+B_{i} G_{n_{i}}(x)\right](i=1,2) .
$$

\subsection{Lemma 3}

$e^{-\frac{1}{2} x^{2}} H_{n_{i}}(x), e^{-\frac{1}{2} x^{2}} G_{n_{i}}(x)$ are two linear independent solutions to the first Weber equations $z_{i}^{\prime \prime}+\left(2 n_{i}+1-x^{2}\right) z_{i}=0(i=1,2) \quad$. Defining functions of guide solution as follows:

$$
\begin{aligned}
& \varphi_{0,0}^{i}(x, \xi)=e^{-\frac{1}{2}\left(x^{2}+\xi^{2}\right)}\left[H_{n_{i}}(x) G_{n_{i}}(\xi)-G_{n_{i}}(x) H_{n_{i}}(\xi)\right](4) \\
& \varphi_{1,0}^{i}(x, \xi)=\frac{\partial}{\partial x} \varphi_{0,0}^{i}(x, \xi) \\
& =-x \varphi_{0,0}^{i}(x, \xi) \\
& \quad+e^{-\frac{1}{2}\left(x^{2}+\xi^{2}\right)}\left[2 n_{i} H_{n_{i}-1}(x) G_{n_{i}}(\xi)-G_{n_{i}-1}(x) H_{n_{i}}(\xi)\right] \\
& \varphi_{0,1}^{i}(x, \xi)=\frac{\partial}{\partial \xi} \varphi_{0,0}^{i}(x, \xi) \\
& =-\xi \varphi_{0,0}^{i}(x, \xi) \\
& \quad+e^{-\frac{1}{2}\left(x^{2}+\xi^{2}\right)}\left[H_{n_{i}}(x) G_{n_{i}-1}(\xi)-2 n_{i} G_{n_{i}}(x) H_{n_{i}-1}(\xi)\right] \\
& \varphi_{1,1}^{i}(x, \xi)=\frac{\partial^{2}}{\partial x \partial \xi} \varphi_{0,0}^{i}(x, \xi) \\
& =x \xi \varphi_{0,0}^{i}(x, \xi) \\
& -x e^{-\frac{1}{2}\left(x^{2}+\xi^{2}\right)}\left[H_{n_{i}}(x) G_{n_{i}-1}(\xi)-2 n_{i} G_{n_{i}}(x) H_{n_{i}-1}(\xi)\right] \\
& -\xi e^{-\frac{1}{2}\left(x^{2}+\xi^{2}\right)}\left[2 n_{i} H_{n_{i}-1}(x) G_{n_{i}}(\xi)-G_{n_{i}-1}(x) H_{n_{i}}(\xi)\right] \\
& +e^{-\frac{1}{2}\left(x^{2}+\xi^{2}\right)}\left[2 n_{i} H_{n_{i}-1}(x) G_{n_{i}-1}(\xi)-2 n_{i} G_{n_{i}-1}(x) H_{n_{i}-1}(\xi)\right]
\end{aligned}
$$

where $i=1$ denotes left region $(a \leq x \leq c), i=2$ denotes right region $(c \leq x \leq b)$.

\section{The Main Theorem and Its Proof}

\subsection{Theorem}

If the boundary value problem (1) has unique solution, then the solution of left region is expressed as:

$$
z_{1}=D \cdot \frac{1}{E+\frac{1}{F+\Phi_{1}(a)}} \cdot \frac{1}{F+\Phi_{1}(a)} \cdot \Phi_{1}(x) \quad(a \leq x \leq c)(8)
$$

and the solution of right region is expressed as: 


$$
\begin{aligned}
z_{2}= & D \cdot \frac{1}{E+\frac{1}{F+\Phi_{1}(a)} \cdot \frac{1}{F+\Phi_{1}(a)}} \\
& \cdot \frac{\varphi_{0,1}^{1}(c, c)}{\lambda \Phi_{2}(c) \varphi_{1,1}^{1}(\mathrm{a}, c)-\mu \varphi_{1,0}^{1}(\mathrm{a}, c)} \cdot \Phi_{2}(x) \quad(c \leq x \leq b)
\end{aligned}
$$

where $\Phi_{2}(x)$ is called the similar kernel function of right region:

$$
\Phi_{2}(x)=\frac{M \varphi_{0,0}^{2}(x, b)+N \varphi_{0,1}^{2}(x, b)}{M \varphi_{1,0}^{2}(\mathrm{c}, b)+N \varphi_{1,1}^{2}(\mathrm{c}, b)} \quad(c \leq x \leq b)
$$

and $\Phi_{1}(x)$ is called the similar kernel function of left region:

$$
\Phi_{1}(x)=\frac{\lambda \Phi_{2}(c) \varphi_{0,1}^{1}(x, \mathrm{c})-\mu \varphi_{0,0}^{1}(x, \mathrm{c})}{\lambda \Phi_{2}(c) \varphi_{1,1}^{1}(\mathrm{a}, \mathrm{c})-\mu \varphi_{1,0}^{1}(\mathrm{a}, \mathrm{c})} \quad(a \leq x \leq c)(11)
$$

\subsubsection{Proof}

According to the lemma 2, we know that general solutions to governing equations of left and right region of the boundary value problem (1) are

$$
z_{i}(x)=e^{-\frac{1}{2} x^{2}}\left[A_{i} H_{n_{i}}(x)+B_{i} G_{n_{i}}(x)\right] \quad(i=1,2)
$$

We calculate derivative of $z_{i}(x)$ to $x$ :

$$
\begin{aligned}
& z_{i}^{\prime}(x)=\frac{d}{d x}\left\{e^{-\frac{1}{2} x^{2}}\left[A_{i} H_{n_{i}}(x)+B_{i} G_{n_{i}}(x)\right]\right\} \\
& =e^{-\frac{1}{2} x^{2}}\left\{\begin{array}{l}
A_{i}\left[-x H_{n_{i}}(x)+2 n_{i} H_{n_{i}-1}(x)\right] \\
+B_{i}\left[-x G_{n_{i}}(x)+G_{n_{i}-1}(x)\right]
\end{array}\right\}
\end{aligned}
$$

By substituting Eqs.(12) and (13) into left and right boundary conditions and two connection conditions of the boundary value problem (1), we obtain the following equations respectively:

$$
\begin{aligned}
& A_{1}\left\{E e^{-\frac{1}{2} a^{2}} H_{n_{1}}(a)+(1+E F) \frac{d}{d x}\left[e^{-\frac{1}{2} x^{2}} H_{n_{1}}(x)\right]_{x=a}\right\} \\
& +B_{1}\left\{E e^{-\frac{1}{2} a^{2}} G_{n_{1}}(a)+(1+E F) \frac{d}{d x}\left[e^{-\frac{1}{2} x^{2}} G_{n_{1}}(x)\right]_{x=a}\right\} \\
& =A_{1}\left\{\begin{array}{l}
E e^{-\frac{1}{2} a^{2}} H_{n_{1}}(a)+(1+E F) e^{-\frac{1}{2} a^{2}} \\
{\left[-a H_{n_{1}}(a)+2 n_{1} H_{n_{1}-1}(a)\right]}
\end{array}\right\} \\
& +B_{1}\left\{\begin{array}{l}
E e^{-\frac{1}{2} a^{2}} G_{n_{1}}(a)+(1+E F) e^{-\frac{1}{2} a^{2}} \\
{\left[-a G_{n_{1}}(a)+G_{n_{1}-1}(a)\right]}
\end{array}\right\}=D
\end{aligned}
$$

$$
\begin{aligned}
& A_{1} e^{-\frac{1}{2} c^{2}} H_{n_{1}}(c)+B_{1} e^{-\frac{1}{2} c^{2}} G_{n_{1}}(c) \\
& -A_{2} \lambda e^{-\frac{1}{2} c^{2}} H_{n_{2}}(c)-B_{2} \lambda e^{-\frac{1}{2} c^{2}} G_{n_{2}}(c)=0 \\
& A_{1} \frac{d}{d x}\left[e^{-\frac{1}{2} x^{2}} H_{n_{1}}(x)\right]_{x=c}+B_{1} \frac{d}{d x}\left[e^{-\frac{1}{2} x^{2}} G_{n_{1}}(x)\right]_{x=c} \\
& -A_{2} \mu \frac{d}{d x}\left[e^{-\frac{1}{2} x^{2}} H_{n_{2}}(x)\right]_{x=c}-B_{2} \mu \frac{d}{d x}\left[e^{-\frac{1}{2} x^{2}} G_{n_{2}}(x)\right]_{x=c} \\
& =A_{1} e^{-\frac{1}{2} c^{2}}\left[-c H_{n_{1}}(c)+2 n_{1} H_{n_{1}-1}(c)\right] \\
& +B_{1} e^{-\frac{1}{2} c^{2}}\left[-c G_{n_{1}}(c)+G_{n_{1}-1}(c)\right] \\
& -A_{2} \mu e^{-\frac{1}{2} c^{2}}\left[-c H_{n_{2}}(c)+2 n_{2} H_{n_{2}-1}(c)\right] \\
& -B_{2} \mu e^{-\frac{1}{2} c^{2}}\left[-c G_{n_{2}}(c)+G_{n_{2}-1}(c)\right]=0 \\
& A_{2}\left\{M e^{-\frac{1}{2} b^{2}} H_{n_{2}}(b)+N \frac{d}{d x}\left[e^{-\frac{1}{2} x^{2}} H_{n_{2}}(x)\right]_{x=b}\right\} \\
& +B_{2}\left\{M e^{-\frac{1}{2} b^{2}} G_{n_{2}}(b)+N \frac{d}{d x}\left[e^{-\frac{1}{2} x^{2}} G_{n_{2}}(x)\right]_{x=b}\right\} \\
& =A_{2}\left\{\begin{array}{l}
M e^{-\frac{1}{2} b^{2}} H_{n_{2}}(b)+N e^{-\frac{1}{2} b^{2}} \\
{\left[-b H_{n_{2}}(b)+2 n_{2} H_{n_{2}-1}(b)\right]}
\end{array}\right\} \\
& +B_{2}\left\{\begin{array}{l}
M e^{-\frac{1}{2} b^{2}} G_{n_{2}}(b)+N e^{-\frac{1}{2} b^{2}} \\
{\left[-b G_{n_{2}}(b)+G_{n_{2}-1}(b)\right]}
\end{array}\right\}=0
\end{aligned}
$$

According to the uniqueness of solution to the boundary value problem (1), we know that the coefficient determinant $\Delta$ of linear system (Eqs.(14) (17)) about undetermined coefficients is not equal to zero, and

$$
\begin{array}{r}
\Delta=E\left[\begin{array}{c}
-M \mu \varphi_{1,0}^{2}(c, b) \varphi_{0,0}^{1}(a, c) \\
+M \lambda \varphi_{0,0}^{2}(c, b) \varphi_{0,1}^{1}(a, c) \\
-N \mu \varphi_{1,1}^{2}(c, b) \varphi_{0,0}^{1}(a, c) \\
+N \lambda \varphi_{0,1}^{2}(c, b) \varphi_{0,1}^{1}(a, c)
\end{array}\right] \\
(1+E F)\left[\begin{array}{l}
-M \mu \varphi_{1,0}^{2}(c, b) \varphi_{1,0}^{1}(a, c) \\
+M \lambda \varphi_{0,0}^{2}(c, b) \varphi_{1,1}^{1}(a, c) \\
-N \mu \varphi_{1,1}^{2}(c, b) \varphi_{1,0}^{1}(a, c) \\
+N \lambda \varphi_{0,1}^{2}(c, b) \varphi_{1,1}^{1}(a, c)
\end{array}\right]
\end{array}
$$

Values of $A_{1}, B_{1}, A_{2}, B_{2}$ can be obtained by using the Cramer rule as follows: 


$$
\begin{aligned}
& A_{1}=\frac{D}{\Delta}\left\{\begin{array}{l}
-M \mu e^{-\frac{1}{2} c^{2}} G_{n_{1}}(c) \varphi_{1,0}^{2}(c, b) \\
+M \lambda e^{-\frac{1}{2} c^{2}} \varphi_{0,0}^{2}(c, b)\left[-c G_{n_{1}}(c)+G_{n_{1}-1}(c)\right] \\
-N \mu e^{-\frac{1}{2} c^{2}} G_{n_{1}}(c) \varphi_{1,1}^{2}(c, b) \\
+N \lambda e^{-\frac{1}{2} c^{2}} \varphi_{0,1}^{2}(c, b)\left[-c G_{n_{1}}(c)+G_{n_{1}-1}(c)\right]
\end{array}\right\} \\
& B_{1}=-\frac{D}{\Delta}\left\{\begin{array}{l}
-M \mu e^{-\frac{1}{2} c^{2}} H_{n_{1}}(c) \varphi_{1,0}^{2}(c, b) \\
+M \lambda e^{-\frac{1}{2} c^{2}} \varphi_{0,0}^{2}(c, b)\left[-c H_{n_{1}}(c)+2 n_{1} H_{n_{1}-1}(c)\right] \\
-N \mu e^{-\frac{1}{2} c^{2}} H_{n_{1}}(c) \varphi_{1,1}^{2}(c, b) \\
+N \lambda e^{-\frac{1}{2} c^{2}} \varphi_{0,1}^{2}(c, b)\left[-c H_{n_{1}}(c)+2 n_{1} H_{n_{1}-1}(c)\right]
\end{array}\right\}
\end{aligned}
$$

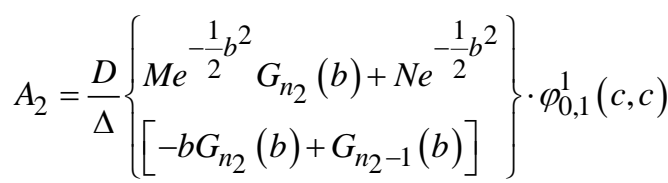

$$
\begin{aligned}
& B_{2}=-\frac{D}{\Delta}\left\{\begin{array}{l}
M e^{-\frac{1}{2} b^{2}} H_{n_{2}}(b)+N e^{-\frac{1}{2} b^{2}} \\
{\left[\begin{array}{l}
-b H_{n_{2}}(b)+2 n_{2} H_{n_{2}-1}(b)
\end{array}\right]}
\end{array}\right\} \cdot \varphi_{0,1}^{1}(c, c)(22)
\end{aligned}
$$

By substituting values of $A_{1}, B_{1}, A_{2}, B_{2}$ (19)-(22) into Eq.(12) and using the similar kernel function of right region Eq.(10) and the similar kernel function of left region Eq.(11), solutions of left and right regions to the boundary value problem (1) are obtained respectively. i.e. Eq.(8) and Eq.(9).

\subsubsection{Corollary 1}

In the boundary value problem (1), if the right boundary condition is $z_{2}(b)=0$ (i.e. $M \neq 0, N=0 \quad$ ), the corresponding similar kernel function of right region is

$$
\Phi_{2}(x)=\frac{\varphi_{0,0}^{2}(x, b)}{\varphi_{1,0}^{2}(c, b)} .
$$

\subsubsection{Corollary 2}

In the boundary value problem (1), if the right boundary condition is $z_{2}^{\prime}(b)=0$ (i.e. $M=0, N \neq 0 \quad$ ), the corresponding similar kernel function of right region is

$$
\Phi_{2}(x)=\frac{\varphi_{0,1}^{2}(x, b)}{\varphi_{1,1}^{2}(c, b)} .
$$

\subsubsection{Corollary 3}

The first continued fraction, which belongs to the structure of the solution (i.e. Eq. (8)) to the boundary value problem (1), has the following property:

$$
\left[z_{1}(x)+F z_{1}^{\prime}(x)\right]_{x=a}=\frac{D}{E+\frac{1}{F+\Phi_{1}(a)}}
$$

\section{Steps of the SCM}

According to the proof of lemma 2 and theorem 1, it is easy to induce steps of the SCM for solving the boundary value problem of the composite first Weber system. The concrete steps are as follows:

\subsection{Step 1 Constructing Functions of Guide Solution}

we structure the function of guide solution of left region by using two linear independent solutions $e^{-\frac{1}{2} x^{2}} H_{n_{1}}(x)$ and $e^{-\frac{1}{2} x^{2}} G_{n_{1}}(x)$ to the governing equation of the left region of the boundary value problem (1) and structure the function of guide solution of right region by using two linear independent solutions $e^{-\frac{1}{2} x^{2}} H_{n_{2}}(x)$ and $e^{-\frac{1}{2} x^{2}} G_{n_{2}}(x)$ to the governing equation of the left region of the boundary value problem (1) as follows: $\varphi_{0,0}^{i}(x, \xi)(i=1,2)$. Other functions of guide solution can be obtained by calculating partial derivatives of $\varphi_{0,0}^{i}(x, \xi)$ to $x, \xi$ respectively.

\subsection{Step 2 Constructing Similar Kernel Functions of Left and Right Regions}

The similar kernel function $\Phi_{2}(x)$ of right region of the boundary value problem (1) can be structured by using functions of guide solution of right region and coefficients $M, N$ of the homogeneous right boundary condition, as shown Eq.(10). Further we calculate $\Phi_{2}(c)$.The similar kernel function $\Phi_{1}(x)$ of left region of the boundary value problem (1) can be structured by using functions of guide solution of left region, coefficients $\lambda, \mu$ of two connection conditions and $\Phi_{2}(c)$, as shown Eq.(11). Further we calculate $\Phi_{1}(a)$.

\subsection{Step 3 Obtaining Solutions to the Boundary Value Problem}

To the boundary value problem (1), the solution of the left region can be obtained by assembling coefficients $D, E, F$ of the non-homogeneous left boundary condition, the similar kernel function $\Phi_{1}(x)$ of left region and $\Phi_{1}(a)$, as shown Eq.(8). The solution of the right region can be obtained by assembling the coefficients $D, E, F$ of the non-homogeneous left boundary condition, the function of guide solution of left region, coefficients $\lambda, \mu$ of two connection conditions, the similar kernel function $\Phi_{2}(x)$ of right region, $\Phi_{2}(c)$ and $\Phi_{1}(a)$, as shown Eq.(9). 


\section{The Application of the SCM}

Solving the boundary value problem as follows:

$$
\left\{\begin{array}{l}
z_{1}^{\prime \prime}+\left(3-x^{2}\right) z_{1}=0 \\
z_{2}^{\prime \prime}+\left(5-x^{2}\right) z_{2}=0 \\
\left.z_{1}^{\prime}\right|_{X=0}=1 \\
\left.z_{1}\right|_{X=1}=\left.z_{2}\right|_{X=1} \\
\left.z_{1}^{\prime}\right|_{X=1}=\left.2 z_{2}^{\prime}\right|_{X=1} \\
\left.2 z_{2}^{\prime}\right|_{X=2}=0
\end{array}\right.
$$

Comparing with the boundary value problem (1) and (24), we know that $n_{1}=1, \quad n_{2}=2, a=0, b=2, c=1$, $\lambda=1, \quad \mu=2, \quad D=1, \quad E=0, \quad M=0, \quad N=2$. Governing equations of left and right regions of the boundary value problem (24) are the first Weber equation, then two linear independent solutions of the left governing equation are $e^{-\frac{1}{2} x^{2}} H_{1}(x)$ and $e^{-\frac{1}{2} x^{2}} G_{1}(x)$, and two linear independent solutions of the right governing equation are $e^{-\frac{1}{2} x^{2}} H_{2}(x)$ and $e^{-\frac{1}{2} x^{2}} G_{2}(x)$. According to steps of the SCM, we solve the boundary value problem (24).

\subsection{Step 1 Constructing Functions of Guide Solution}

According to Eqs.(4) (7), we structure functions of guide solution of left and right regions as follows:

$$
\begin{aligned}
& \varphi_{0,0}^{1}(x, \xi)=e^{-\frac{1}{2}\left(x^{2}+\xi^{2}\right)}\left[H_{1}(x) G_{1}(\xi)-G_{1}(x) H_{1}(\xi)\right], \\
& \varphi_{0,0}^{2}(x, \xi)=e^{-\frac{1}{2}\left(x^{2}+\xi^{2}\right)}\left[H_{2}(x) G_{2}(\xi)-G_{2}(x) H_{2}(\xi)\right], \\
& \varphi_{1,0}^{1}(x, \xi)=\frac{\partial}{\partial x} \varphi_{0,0}^{1}(x, \xi) \\
& =-x \varphi_{0,0}^{1}(x, \xi)+e^{-\frac{1}{2}\left(x^{2}+\xi^{2}\right)}, \\
& {\left[2 H_{0}(x) G_{1}(\xi)-G_{0}(x) H_{1}(\xi)\right]} \\
& \varphi_{1,0}^{2}(x, \xi)=\frac{\partial}{\partial x} \varphi_{0,0}^{2}(x, \xi) \\
& =-x \varphi_{0,0}^{2}(x, \xi)+e^{-\frac{1}{2}\left(x^{2}+\xi^{2}\right)}, \\
& {\left[4 H_{1}(x) G_{2}(\xi)-G_{1}(x) H_{2}(\xi)\right]} \\
& \varphi_{0,1}^{1}(x, \xi)=\frac{\partial}{\partial \xi} \varphi_{0,0}^{1}(x, \xi) \\
& =-\xi \varphi_{0,0}^{1}(x, \xi)+e^{-\frac{1}{2}\left(x^{2}+\xi^{2}\right)} \\
& {\left[H_{1}(x) G_{0}(\xi)-2 G_{1}(x) H_{0}(\xi)\right]}
\end{aligned}
$$

$$
\begin{aligned}
\varphi_{0,1}^{2}(x, \xi) & =\frac{\partial}{\partial \xi} \varphi_{0,0}^{2}(x, \xi) \\
& =-\xi \varphi_{0,0}^{2}(x, \xi)+e^{-\frac{1}{2}\left(x^{2}+\xi^{2}\right)} \\
& {\left[H_{2}(x) G_{1}(\xi)-4 G_{2}(x) H_{1}(\xi)\right] } \\
\varphi_{1,1}^{1}(x, \xi) & =\frac{\partial^{2}}{\partial x \partial \xi} \varphi_{0,0}^{1}(x, \xi) \\
& =x \xi \varphi_{0,0}^{1}(x, \xi) \\
& -x e^{-\frac{1}{2}\left(x^{2}+\xi^{2}\right)}\left[H_{1}(x) G_{0}(\xi)-2 G_{1}(x) H_{0}(\xi)\right], \\
& -\xi e^{-\frac{1}{2}\left(x^{2}+\xi^{2}\right)}\left[2 H_{0}(x) G_{1}(\xi)-G_{0}(x) H_{1}(\xi)\right] \\
& +e^{-\frac{1}{2}\left(x^{2}+\xi^{2}\right)}\left[2 H_{0}(x) G_{0}(\xi)-2 G_{0}(x) H_{0}(\xi)\right] \\
& +\xi e^{-\frac{1}{2}\left(x^{2}+\xi^{2}\right)}\left[4 H_{1}(x) G_{2}(\xi)-G_{1}(x) H_{2}(\xi)\right] \\
\varphi_{1,1}^{2}(x, \xi) & =\frac{\partial^{2}}{\partial x \partial \xi} \varphi_{0,0}^{2}(x, \xi) \\
& =x \xi \varphi_{0,0}^{2}(x, \xi) \\
& -\frac{1}{2}\left(x^{2}+\xi^{2}\right) \\
&
\end{aligned}
$$

\subsection{Step 2 Constructing Similar Kernel Functions of Left and Right Regions}

According to the Eq.(10), we structure the similar kernel function of right region of the boundary value problem (24) as follows:

$$
\begin{aligned}
\Phi_{2}(x)= & \left\{\begin{array}{l}
-2 e^{-\frac{1}{2}\left(x^{2}+4\right)}\left[H_{2}(x) G_{2}(2)-G_{2}(x) H_{2}(x)\right] \\
+e^{-\frac{1}{2}\left(x^{2}+4\right)}\left[H_{2}(x) G_{1}(2)-4 G_{2}(x) H_{1}(2)\right]
\end{array}\right\} \\
& \times\left\{\begin{array}{l}
2 e^{-\frac{5}{2}}\left[H_{2}(1) G_{2}(2)-G_{2}(1) H_{2}(2)\right] \\
-e^{-\frac{5}{2}}\left[H_{2}(1) G_{1}(2)-4 G_{2}(1) H_{1}(2)\right] \\
-2 e^{-\frac{5}{2}}\left[4 H_{1}(1) G_{2}(2)-G_{1}(1) H_{2}(2)\right] \\
+e^{-\frac{5}{2}}\left[4 H_{1}(1) G_{1}(2)-4 G_{1}(1) H_{1}(2)\right]
\end{array}\right\}
\end{aligned}
$$

Since

$$
\begin{aligned}
& H_{1}(x)=2 x, H_{2}(x)=2\left(2 x^{2}-1\right), \\
& G_{1}(x)=\sum_{n=0}^{\infty} \frac{x^{2 n+2}}{n !(2 n+1)}-\frac{1}{2} e^{x^{2}},
\end{aligned}
$$




$$
G_{2}(x)=\frac{2 x^{2}-1}{4} \sum_{n=0}^{\infty} \frac{x^{2 n+1}}{n !(2 n+1)}-\frac{x e^{x^{2}}}{4}[27]
$$

so

$$
\Phi_{2}(x)=e^{-\frac{1}{2}\left(x^{2}-1\right)}\left[\begin{array}{l}
-7\left(2 x^{2}-1\right) \sum_{n=0}^{\infty} \frac{2^{2 n+1}}{n !(2 n+1)} \\
+3\left(2 x^{2}-1\right) \sum_{n=0}^{\infty} \frac{x^{2 n+1}}{n !(2 n+1)} \\
+2\left(2 x^{2}-1\right) \sum_{n=0}^{\infty} \frac{2^{2 n+2}}{n !(2 n+1)} \\
-3 x e^{x^{2}}+\left(2 x^{2}-1\right) e^{4}
\end{array}\right]
$$$$
\times\left[\begin{array}{l}
-21 \sum_{n=0}^{\infty} \frac{2^{2 n+1}}{n !(2 n+1)}+9 \sum_{n=0}^{\infty} \frac{1}{n !(2 n+1)} \\
+6 \sum_{n=0}^{\infty} \frac{2^{2 n+2}}{n !(2 n+1)}+3 e^{4}-3 e
\end{array}\right]^{-1}
$$

$(1 \leq x \leq 2)$

Let $x=1$, then

$$
\begin{aligned}
& \Phi_{2}(1)=\left[\begin{array}{l}
-7 \sum_{n=0}^{\infty} \frac{2^{2 n+1}}{n !(2 n+1)}+3 \sum_{n=0}^{\infty} \frac{1}{n !(2 n+1)} \\
+2 \sum_{n=0}^{\infty} \frac{2^{2 n+2}}{n !(2 n+1)}+e^{4}-3 e
\end{array}\right] \\
& \times\left[\begin{array}{l}
-21 \sum_{n=0}^{\infty} \frac{2^{2 n+1}}{n !(2 n+1)} \\
+9 \sum_{n=0}^{\infty} \frac{1}{n !(2 n+1)}+6 \sum_{n=0}^{\infty} \frac{2^{2 n+2}}{n !(2 n+1)}+3 e^{4}-3 e
\end{array}\right]
\end{aligned}
$$

Then according to the Eq. (11), we structure the left similar kernel function of the boundary value problem (24) as follows:

$$
\begin{aligned}
& \left\{\begin{array}{l}
\Phi_{2}(1)\left\{\begin{array}{l}
-e^{-\frac{1}{2}\left(x^{2}+1\right)}\left[H_{1}(x) G_{1}(1)-G_{1}(x) H_{1}(1)\right] \\
+e^{-\frac{1}{2}\left(x^{2}+1\right)}\left[H_{1}(x) G_{0}(1)-2 G_{1}(x) H_{0}(1)\right]
\end{array}\right\} \\
-2 e^{-\frac{1}{2}\left(x^{2}+1\right)}\left[H_{1}(x) G_{1}(1)-G_{1}(x) H_{1}(1)\right]
\end{array}\right\} \\
& \times\left\{\begin{array}{l}
\Phi_{2}(1)\left\{\begin{array}{l}
-e^{-\frac{1}{2}}\left[2 H_{0}(0) G_{1}(1)-G_{0}(0) H_{1}(1)\right]+ \\
e^{-\frac{1}{2}}\left[2 H_{0}(0) G_{0}(1)-2 G_{0}(0) H_{0}(1)\right]
\end{array}\right\} \\
-2 e^{-\frac{1}{2}}\left[2 H_{0}(0) G_{1}(1)-G_{0}(0) H_{1}(1)\right]
\end{array}\right\}
\end{aligned}
$$

Since

$$
\begin{aligned}
& H_{0}(x)=1, H_{1}(x)=2 x, \\
& G_{0}(x)=\sum_{n=0}^{\infty} \frac{x^{2 n+1}}{n !(2 n+1)}, \\
& G_{1}(x)=\sum_{n=0}^{\infty} \frac{x^{2 n+2}}{n !(2 n+1)}-\frac{1}{2} e^{x^{2}}
\end{aligned}
$$

So

$$
\begin{aligned}
& \Phi_{1}(x)=e^{-\frac{x^{2}}{2}}\left\{\begin{array}{l}
\Phi_{2}(1)\left[-4 \sum_{n=0}^{\infty} \frac{x^{2 n+1}}{n !(2 n+1)}+2 e^{x^{2}}+e x\right. \\
-4 x \sum_{n=0}^{\infty} \frac{1}{n !(2 n+1)} \\
-2 \sum_{n=0}^{\infty} \frac{x^{2 n+2}}{n !(2 n+1)}+e^{x^{2}}+2 e x
\end{array}\right\} \\
& \times\left[\Phi_{2}(1) e-4 \sum_{n=0}^{\infty} \frac{1}{n !(2 n+1)}+2 e\right]^{-1}(0 \leq x \leq 1)
\end{aligned}
$$

Let $x=0$, then

$$
\Phi_{1}(0)=\frac{2 \Phi_{2}(1)+1}{\Phi_{2}(1) e-4 \sum_{n=0}^{\infty} \frac{1}{n !(2 n+1)}+2 e} .
$$

\subsection{Step 3 Obtaining Solutions of The Boundary Value Problem (24)}

According to Eqs.(8) and (9), solutions of left and right regions of the boundary value problem (24) can be obtained respectively as follows:

$$
z_{1}(x)=\Phi_{1}(x)=e^{-\frac{x^{2}}{2}}\left\{\begin{array}{l}
\Phi_{2}(1)\left[\begin{array}{c}
-4 \sum_{n=0}^{\infty} \frac{x^{2 n+1}}{n !(2 n+1)} \\
+2 e^{x^{2}}+e x
\end{array}\right] \\
-4 x \sum_{n=0}^{\infty} \frac{1}{n !(2 n+1)} \\
-2 \sum_{n=0}^{\infty} \frac{x^{2 n+2}}{n !(2 n+1)}+e^{x^{2}}+2 e x
\end{array}\right\},
$$

$$
\times\left[\Phi_{2}(1) e-4 \sum_{n=0}^{\infty} \frac{1}{n !(2 n+1)}+2 e\right]^{-1}(0 \leq x \leq 1)
$$$$
z_{2}(x)=\left\{\Phi_{2}(x) e^{-1}\left[H_{1}(1) G_{0}(1)-2 G_{1}(1) H_{0}(1)\right]\right\}
$$

$$
\begin{aligned}
& \times\left\{\begin{array}{l}
\Phi_{2}(1)\left\{\begin{array}{c}
-e^{-\frac{1}{2}}\left[2 H_{0}(0) G_{1}(1)-G_{0}(0) H_{1}(1)\right] \\
+e^{-\frac{1}{2}}\left[2 H_{0}(0) G_{0}(1)-2 G_{0}(0) H_{0}(1)\right]
\end{array}\right\} \\
-2 e^{-\frac{1}{2}}\left[2 H_{0}(0) G_{1}(1)-G_{0}(0) H_{1}(1)\right]
\end{array}\right\}^{-1}, \\
& =e^{\frac{1}{2}} \Phi_{2}(x) \times\left[\Phi_{2}(1) e-4 \sum_{n=0}^{\infty} \frac{1}{n !(2 n+1)}+2 e\right]^{-1}(1 \leq x \leq 2)
\end{aligned}
$$

where

$$
\begin{gathered}
H_{0}(x)=1, H_{1}(x)=2 x, \\
H_{2}(x)=2\left(2 x^{2}-1\right), G_{0}(x)=\sum_{n=0}^{\infty} \frac{x^{2 n+1}}{n !(2 n+1)}, \\
G_{1}(x)=\sum_{n=0}^{\infty} \frac{x^{2 n+2}}{n !(2 n+1)}-\frac{1}{2} e^{x^{2}},
\end{gathered}
$$




$$
G_{2}(x)=\frac{2 x^{2}-1}{4} \sum_{n=0}^{\infty} \frac{x^{2 n+1}}{n !(2 n+1)}-\frac{x e^{x^{2}}}{4}[27]
$$

\section{Conclusions and Understanding}

(1) In the process of solving the boundary value problem of the composite first Weber system, we just need to obtain two linear independent solutions to governing equation of left region and two linear independent solutions to governing equation of right region of the boundary value problem respectively. Then according to Steps of the SCM, we can obtain solutions to the boundary value problem. Thus, using SCM can avoid the tedious calculation process.

(2) According to structural equations of similar kernel functions Eqs. (10) and (11) and structural equations of solutions of the boundary value problem (1) Eqs. (8) and (9), we know that we only need to change coefficients of boundary conditions to obtain solutions to the boundary value problem (1), when boundary conditions of the boundary value problem (1) change. Thus, Similar Constructing Method is simple and effective for solving the complicated boundary value problem of differential system.

\section{Acknowledgement}

This works is supported the Natural Science Key Projects of the Sichuan Education Bureau of China (Grant No. 12ZA164) and the by the Innovation Fund of Postgraduate Xihua University (Grant No. Ycjj201310).

\section{References}

[1] LI Shunchu, YI Liangzhong, Zheng Pengshe. The Similar Structure of Differential Equations on Fixed Solution Problem (in Chinese). Journal of Sichuan University (Natural Science Edition), 2006,43(4):933-934.

[2] JIA Minhui, LI Shunchu. The Similar Structure of Solution Differential Equation on Boundary Value Problem (in Chinese). College Mathematics, 2005,21(5):37-39.

[3] LI Shunchu. The Similar Structure of Solution of Second-order Linear Homogeneous Differential Equations with Constant Coefficients on the Boundary Value Problem (in Chinese). Journal of Xihua University (Natural Science Edition), 2007,26(1):84-85.

[4] LI Shunchu. The Similar Structure of Solution to the Boundary Value Problem for Second-order Linear Homogeneous Differential Equations (in Chinese). Journal of Xihua University (Natural Science Edition), 2009,28(5): 40-41(to 90).

[5] Yan Juan, Li Shunchu, Xing Chenglin. The Similar Structures of Solutions to First Class Boundary Value Problem of Sceond-order Euler Equation (in Chinese). Journal of Xihua University (Natural Science Edition), 2009, 28(6):86-88.

[6] LI Quanyong, LI Shunchu, Chi Ying. The Similar Structure of Solution of Linear Homogeneous Differential Equations with Constant Coefficients on the Boundary Value Problem (in Chinese). Sichuan Ordnance Journal, 2010,31(4): 126-129.

[7] Huang Rongjun, Li Shunchu, Xu Dongxu. Similar Constructive Method of Solution to Boundary Value of First Weber Equation (in Chinese). Journal of Mianyang Normal University, 2012, 31(11):1-5(-15).

[8] Li Shunchu, Liao Zhijian. Constructing the Solution of Boundary Value Problem of the Differential Equation with its an Arbitrary Non-trivial Solution (in Chinese). Journal of Sichuan University (Natural Science Edition), 2012, 49(6):1209-1213.
[9] LI Shun-chu. Preliminary Exploration and Prospects of the Similar Structure of Solutions of Differential Equations (in Chinese). Journal of Xihua University (Natural Science Edition), 2010,29(2):223-226(to238).

[10] Tian Jidong, Li Shunchu. The Formal Similarity of Solutions in the Laplace Space on the Class of Quasilinear Partial Differential Equation. MATHEMATICAL THEORY AND APPLICATIONS, 2004, 24(2):66-73.

[11] Jia Minhui, LI Shun-chu. The Formal Similarity of Solutions in the Laplace Space on the Class of Fluid Flow Differential Equation. Journal of Electronic Science and Technology of China, 2005,3(2):172-174.

[12] SU Jian-peng, LI Shun-chu, LI Cheng-jie. The Similar of Solutions in the Laplace Space of Composite Parabolic Partial Differential Equation. Journal of Zaozhuang University, 2009,26(2):6-11.

[13] LI Shun-chu. The Formal Similarity of Solutions in the Laplace Space on the Class of Partial Differential Equation System (in English). Journal of Xihua University (Natural Science Edition), 2007,26(4):83-86.

[14] LI Shun-chu, ZHENG Peng-she, ZHANG Yu-fei. The Similar Structure of Pressure Distribution in the Homogenous Reservoir (in Chinese). Pure and Applied Mathematics, 2006,22(4):459-463.

[15] XU wen-zhao, LI Shun-chu, ZHENG Peng-she. The Structure of the Solution of Pressure Distribution in the Fractal Homogenous Reservoir and Analytic Graph (in Chinese). Mathematics and its Applications. Beijing: Atomic Energy Publishing Company,2007: 541-544.

[16] LI Shun-chu, ZHENG Peng-she, ZHANG Yu-fei. The Similar Structure of Pressure Distribution in the Composite Reservoir (in Chinese). Journal of Mathematics in Practice and Theory, 2008,38(3):23-28.

[17] LI Shun-chu, ZHENG Peng-she, ZHANG Yu-fei. Similar structure of pressure distribution in the multilayer reservoir (in Chinese). Applied Mathematics A Journal of Chinese Universities, 2009,24(2):234-238.

[18] LI Shun-chu, ZHANG Jian-jun. Similar Structure of Pressure Distribution in the Fractal Dual Porosity Reservoir (in Chinese). Journal of Xihua University (Natural Science Edition), 2006,25(1):40-43.

[19] Xu changxue, Li Shunchu, Zhu Weibing, et al. The Similar Structure of Pressure Distribution in the Dual Porosity Reservoir (in Chinese). Drilling \& Production Technology, 2006,29(4):28-30.

[20] Xu changxue, Li Shunchu, Zhu Weibing. The Similar Structure of Pressure Distribution in the Fractal Composite Reservoir (in Chinese). Drilling \& Production Technology,2006,29(5):39-42.

[21] Zheng Pengshe, Li Shunchu, Zhang Yufei. The Formal Similarity of Solutions on the Class of Ordinary Differential Equation System (in Chinese)[J]. Journal of Jilin University (Information Science Edition), 2005,23(8):56-60.

[22] Zhu Weibing, Li Shunchu, Xu changxue. The Similar Structure of Pressure Distribution in the Fractal Multilayer Reservoir (in Chinese). Drilling \& Production Technology,2008,31(3):67-69(72).

[23] ZHENG Peng-she, LI Shun-chu, XU wen-zhao. WELL ANALYSIS METHOD BASED ON THE SIMILAR STRUCTURE OF PRESSURE DISTRIBUTION IN THE COMPOSITE RESERVOIR (in Chinese). Drilling \& Production Technology,2007,30(3): 49-50(-62).

[24] Xu Li, Li Shunchu, Wang Junchao. Similar Structure of the Solutions to Radial-Spherical Seepage Problem Considering Quadratic Gradient Effect (in Chinese). Journal of Chongqing Technology and Business University (Natural Science Edition), 2011, 28(6):585-589.

[25] Li Wei, Li Xiaoping, Li Shunchu, Li Quanyong. Similar Structure of the Solutions of Mathematical Model for the Nonlinear Flow of Fractal Commingled Oil Reservoirs (in Chinese). Petroleum Geology \& Oilfield Development in Daqing, 2012, 31(6):79-83.

[26] LI Quanyong, LI Shunchu, LI Wei, WANG Junchao. Study of the Nonlinear Fluid Flow Modle in Dual-porosity Media Reservoir Based on Similar Structure. CHINESE JOURNAL OF ENGINEERING MATHEMATICS, 2013, 30(1):123-130.

[27] Liu Shishi, Liu Shida. Special Function. Beijing: China Meteorological Press, 2002. 